\title{
LES AUTOMATES PROGRAMMABLES INDUSTRIELS
}

\section{SOMMAIRE :}

- p. 2 : Historique

- p. 3 : Place de l'API dans les SAP

- p. 5 : Architecture des automates

- p. 7 : Câblage des entrées / sorties d'un automate

- p. 10 : Traitement du programme automate

- p. 12 : Programmation

- p. 13 : Sécurité

- p.14: Les automates et la communication

- p.17 : Critères de choix d'un automate 


\section{Historique :}

Les automates programmables industriels sont apparus à la fin des années soixante, à la demande de l'industrie automobile américaine (GM), qui réclamait plus d'adaptabilité de leurs systèmes de commande.

Les coûts de l'électronique permettant alors de remplacer avantageusement les technologies actuelles.

Avant: utilisation de relais électromagnétiques et de systèmes pneumatiques pour la réalisation des parties commandes

$$
\Rightarrow \text { logique câblée }
$$

Inconvénients : cher, pas de flexibilité, pas de communication possible

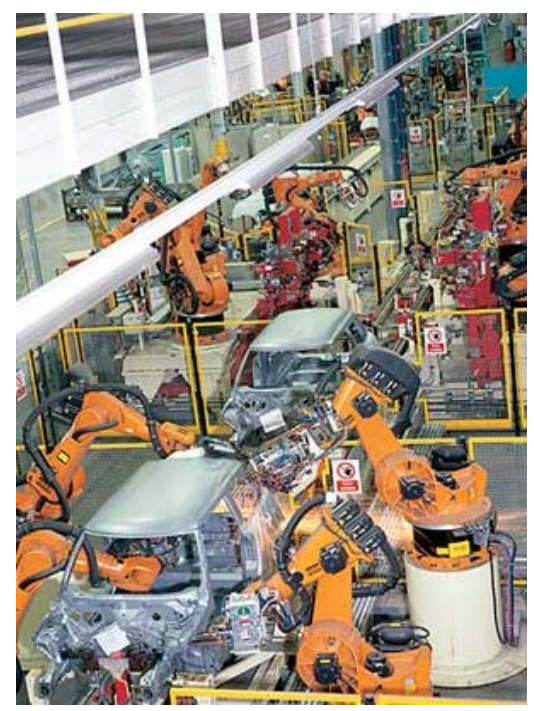

Solution : utilisation de systèmes à base de microprocesseurs permettant une modification aisée des systèmes automatisés $\Rightarrow$ logique programmée

Les ordinateurs de l'époque étant chers et non adaptés aux contraintes du monde industriel, les automates devaient permettre de répondre aux attentes de l'industrie.

\section{Contraintes du monde industriel :}

- influences externes :

- poussières,

- température,

- humidité,

- vibrations,

- parasites électromagnétiques, ...

- Personnel :

- mise en ouvre du matériel aisée (pas de langage de programmation complexe)

- dépannage possible par des techniciens de formation électromécanique

- possibilité de modifier le système en cours de fonctionnement

- Matériel :

- évolutif

- modulaire

- implantation aisée

L'Automate Programmable Industriel (API) est un appareil électronique programmable, adapté à l'environnement industriel, qui réalise des fonctions d'automatisme pour assurer la commande de préactionneurs et d'actionneurs à partir d'informations logique, analogique ou numérique. 


\section{Place de l'API dans le système automatisé de production (S.A.P.) :}

\section{a. Les systèmes automatisés de production :}

L'objectif de l'automatisation des systèmes est de produire, en ayant recours le moins possible à l'homme, des produits de qualité et ce pour un coût le plus faible possible.

Un système automatisé est un ensemble d'éléments en interaction, et organisés dans un but précis : agir sur une matière d'œuvre afin de lui donner une valeur ajoutée.

Le système automatisé est soumis à des contraintes : énergétiques, de configuration, de réglage et d'exploitation qui interviennent dans tous les modes de marche et d'arrêt du système.

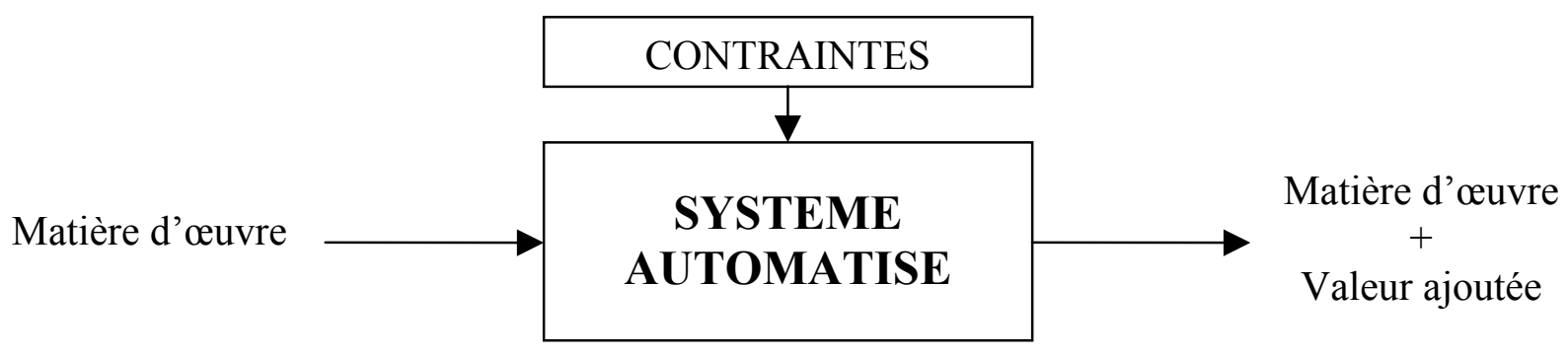

\section{b. Structure d'un système automatisé :}

Tout système automatisé peut se décomposer selon le schéma ci-dessous :

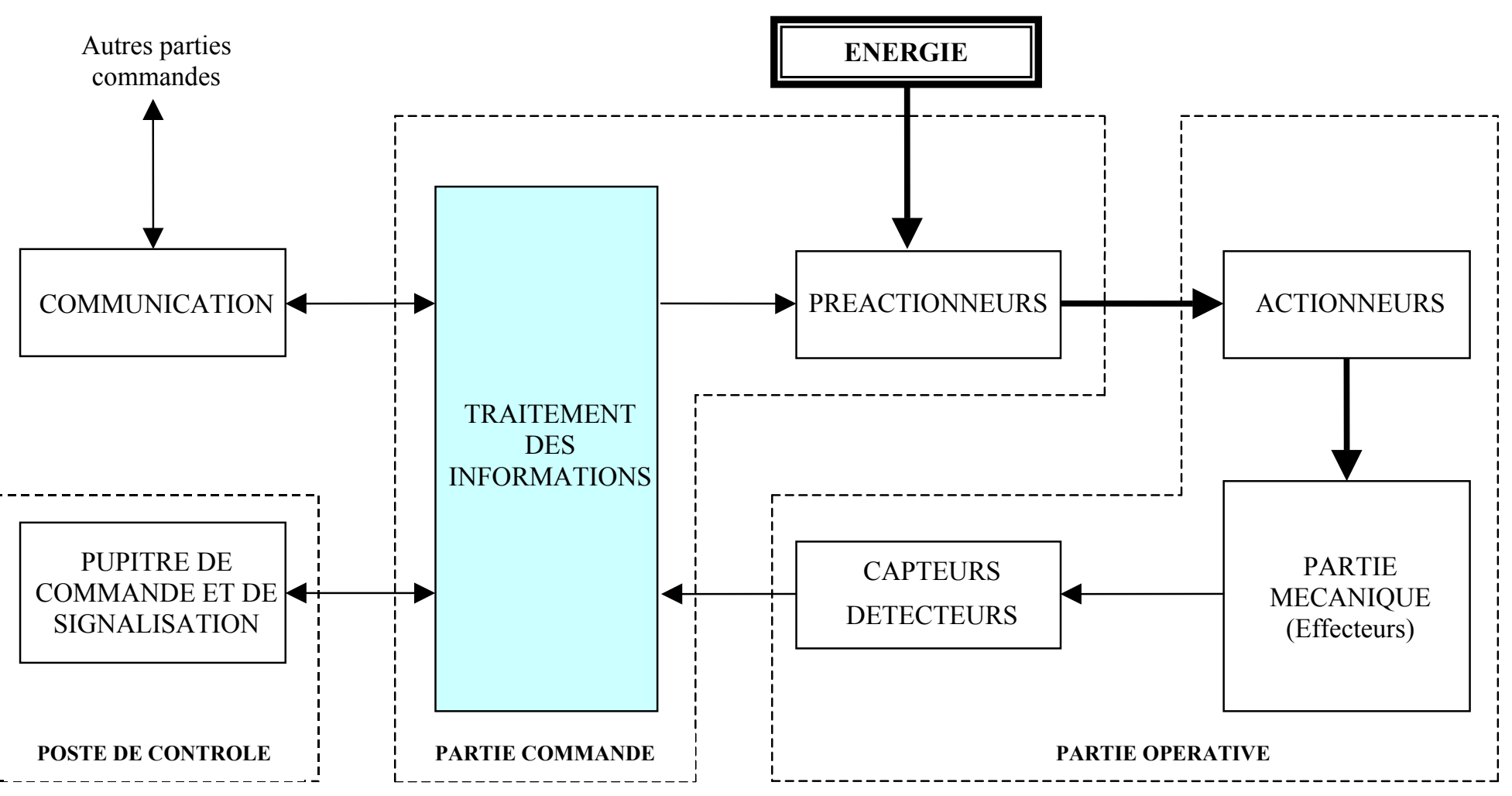




\section{Partie opérative :}

Elle agit sur la matière d'œuvre afin de lui donner sa valeur ajoutée.

Les actionneurs (moteurs, vérins) agissent sur la partie mécanique du système qui agit à son tour sur la matière d'œuvre.

Les capteurs / détecteurs permettent d'acquérir les divers états du système.

\section{Partie commande :}

Elle donne les ordres de fonctionnement à la partie opérative.

Les préactionneurs permettent de commander les actionneurs ; ils assurent le transfert d'énergie entre la source de puissance (réseau électrique, pneumatique ...) et les actionneurs. Exemple : contacteur, distributeur ...

Ces préactionneurs sont commandés à leur tour par le bloc traitement des informations.

Celui-ci reçoit les consignes du pupitre de commande (opérateur) et les informations de la partie opérative transmises par les capteurs / détecteurs.

En fonction de ces consignes et de son programme de gestion des tâches (implanté dans un automate programmable ou réalisé par des relais (on parle de logique câblée)), elle va commander les préactionneurs et renvoyer des informations au pupitre de signalisation ou à d'autres systèmes de commande et/ou de supervision en utilisant un réseau et un protocole de communication.

\section{Poste de contrôle :}

Composé des pupitres de commande et de signalisation, il permet à l'opérateur de commander le système (marche, arrêt, départ cycle ...).

Il permet également de visualiser les différents états du système à l'aide de voyants, de terminal de dialogue ou d'interface homme-machine (IHM).

\section{c. Domaines d'emploi des automates :}

On utilise les API dans tous les secteurs industriels pour la commande des machines (convoyage, emballage ...) ou des chaînes de production (automobile, agroalimentaire ... ) ou il peut également assurer des fonctions de régulation de processus (métallurgie, chimie ...).

Il est de plus en plus utilisé dans le domaine du bâtiment (tertiaire et industriel) pour le contrôle du chauffage, de l'éclairage, de la sécurité ou des alarmes.

\section{d. Nature des informations traitées par l'automate :}

Les informations peuvent être de type :

- $\quad$ Tout ou rien (T.O.R.) : l'information ne peut prendre que deux états (vrai/faux, 0 ou $1 \ldots$ ). C'est le type d'information délivrée par un détecteur, un bouton poussoir ...

- Analogique : l'information est continue et peut prendre une valeur comprise dans une plage bien déterminée. C'est le type d'information délivrée par un capteur (pression, température ...)

- Numérique : l'information est contenue dans des mots codés sous forme binaire ou bien hexadécimale. C'est le type d'information délivrée par un ordinateur ou un module intelligent. 


\section{Architecture des automates :}

\section{a. Aspect extérieur :}

Les automates peuvent être de type compact ou modulaire.

De type compact, on distinguera les modules de programmation (LOGO de Siemens, ZELIO de Schneider, MILLENIUM de Crouzet ...) des microautomates.

Il intègre le processeur, l'alimentation, les entrées et les sorties. Selon les modèles et les fabricants, il pourra réaliser certaines fonctions supplémentaires (comptage rapide, E/S analogiques ...) et recevoir des extensions en nombre limité.

Ces automates, de fonctionnement simple, sont généralement destinés à la commande de petits automatismes.

De type modulaire, le processeur, l'alimentation et les interfaces d'entrées / sorties résident dans des unités séparées (modules) et sont fixées sur un ou plusieurs racks contenant le "fond de panier" (bus plus connecteurs).

Ces automates sont intégrés dans les automatismes complexes où puissance, capacité de traitement et flexibilité sont nécessaires.

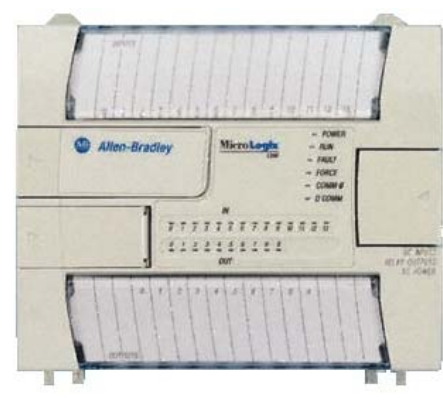

Automate compact (Allen-bradley)

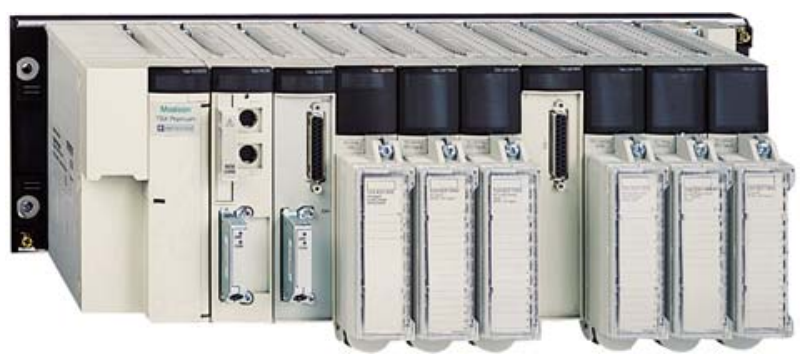

Automate modulaire (Modicon)

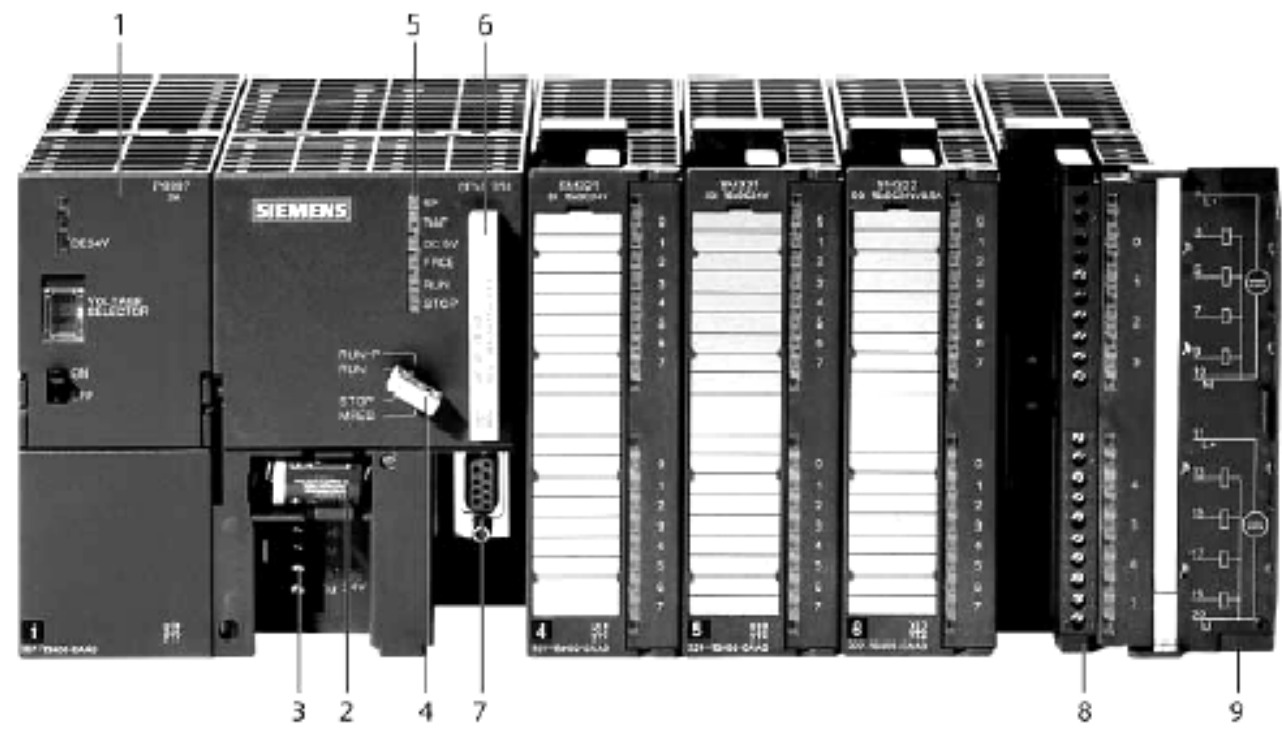

Automate modulaire (Siemens)

1 Module d'alimentation

2 Pile de sauvegarde

3 Connexion au 24V cc

4 Commutateur de mode (à clé)

5 LED de signalisation d'état et de défauts
6 Carte mémoire

7 Interface multipoint (MPI)

8 Connecteur frontal

9 Volet en face avant 


\section{b. Structure interne :}

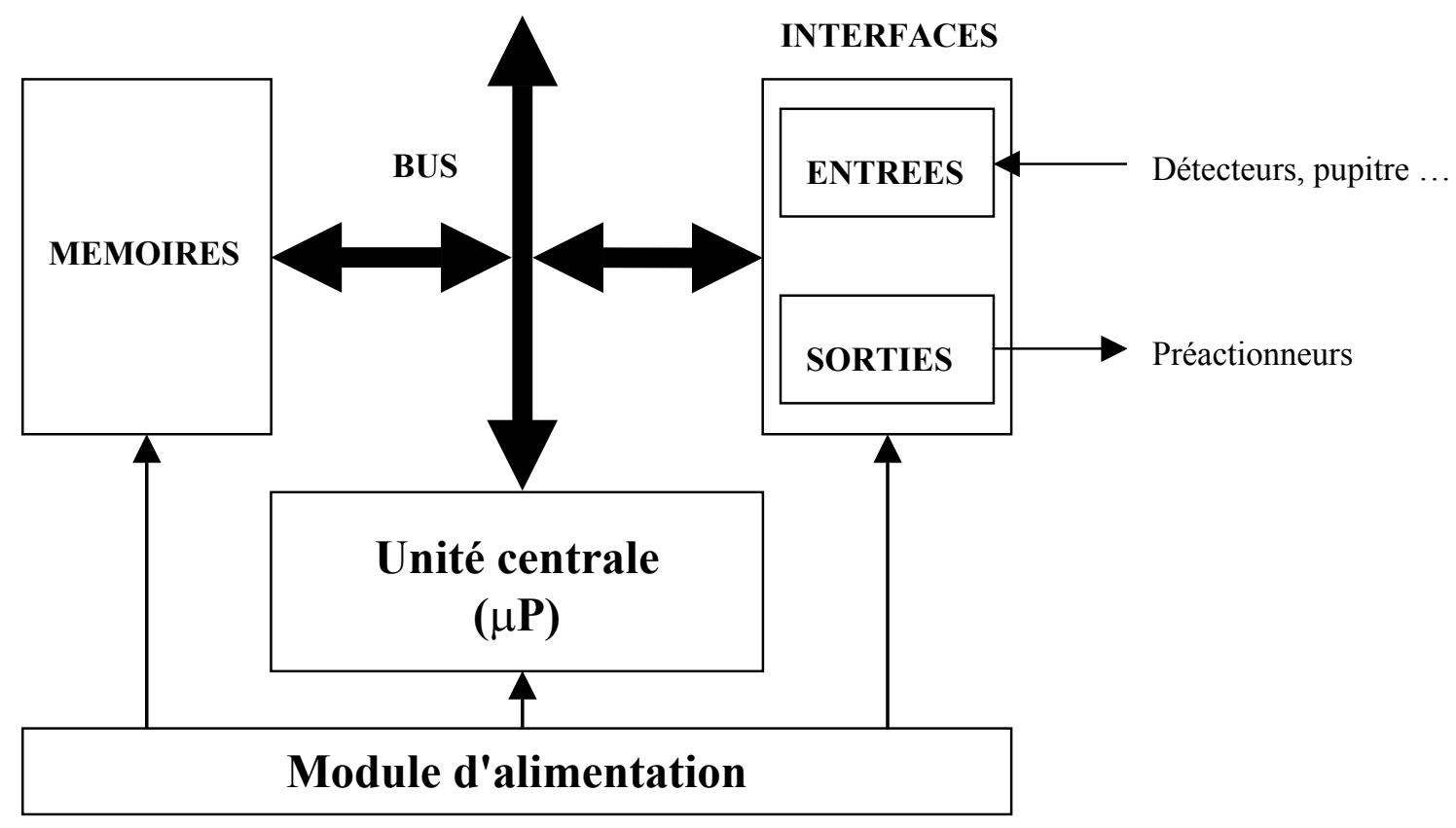

Module d'alimentation : il assure la distribution d'énergie aux différents modules.

Unité centrale : à base de microprocesseur, elle réalise toutes les fonctions logiques, arithmétiques et de traitement numérique (transfert, comptage, temporisation ...).

Le bus interne : il permet la communication de l'ensemble des blocs de l'automate et des éventuelles extensions.

Mémoires : Elles permettent de stocker le système d'exploitation (ROM ou PROM), le programme (EEPROM) et les données système lors du fonctionnement (RAM). Cette dernière est généralement secourue par pile ou batterie. On peut, en règle générale, augmenter la capacité mémoire par adjonction de barrettes mémoires type PCMCIA.

Interfaces d'entrées / sorties :

- Interface d'entrée : elle permet de recevoir les informations du S.A.P. ou du pupitre et de mettre en forme (filtrage, ...) ce signal tout en l'isolant électriquement (optocouplage).

- Interface de sortie : elle permet de commander les divers préactionneurs et éléments de signalisation du S.A.P. tout en assurant l'isolement électrique.

\section{c. Fonctions réalisées :}

Les automates compacts permettent de commander des sorties en T.O.R et gèrent parfois des fonctions de comptage et de traitement analogique.

Les automates modulaires permettent de réaliser de nombreuses autres fonctions grâce à des modules intelligents que l'on dispose sur un ou plusieurs racks. Ces modules ont l'avantage de ne pas surcharger le travail de la CPU car ils disposent bien souvent de leur propre processeur. 


\section{Principales fonctions :}

- Cartes d'entrées / sorties : Au nombre de 4, 8, 16 ou 32, elles peuvent aussi bien réaliser des fonctions d'entrées, de sorties ou les deux.

Ce sont les plus utilisées et les tensions disponibles sont normalisées $(24,48,110$ ou $230 \mathrm{~V}$ continu ou alternatif ...).

Les voies peuvent être indépendantes ou posséder des "communs".

Les cartes d'entrées permettent de recueillir l'information des capteurs, boutons ... qui lui sont raccordés et de la matérialiser par un bit image de l'état du capteur.

Les cartes de sorties offrent deux types de technologies : les sorties à relais électromagnétiques (bobine plus contact ) et les sorties statiques (à base de transistors ou de triacs).

- Cartes de comptage rapide : elles permettent d'acquérir des informations de fréquences élevées incompatibles avec le temps de traitement de l'automate.

Exemple : signal issu d'un codeur de position.

- Cartes de commande d'axe : Elles permettent d'assurer le positionnement avec précision d'élément mécanique selon un ou plusieurs axes. La carte permet par exemple de piloter un servomoteur et de recevoir les informations de positionnement par un codeur. L'asservissement de position pouvant être réalisé en boucle fermée.

- Cartes d'entrées / sorties analogiques : Elles permettent de réaliser l'acquisition d'un signal analogique et sa conversion numérique (CAN) indispensable pour assurer un traitement par le microprocesseur. La fonction inverse (sortie analogique) est également réalisée.

Les grandeurs analogique sont normalisées : 0-10V ou 4-20mA.

- Autres cartes :

Cartes de régulation PID

Cartes de pesage

Cartes de communication (Ethernet ...)

Cartes d'entrées / sorties déportées

\section{Câblage des entrées / sorties d'un automate :}

\section{a. Alimentation de l'automate (voir schéma ci-après) :}

L'automate est alimenté généralement par le réseau monophasé $230 \mathrm{~V} ; 50 \mathrm{~Hz}$ mais d'autres alimentations sont possibles (110V etc ...).

La protection sera de type magnéto-thermique (voir les caractéristiques de l'automate et les préconisations du constructeur).

Il est souhaitable d'asservir l'alimentation de l'automate par un circuit de commande spécifique (contacteur KM1).

De même, les sorties seront asservies au circuit de commande et alimentées après validation du chien de garde (voir paragraphe VII). 


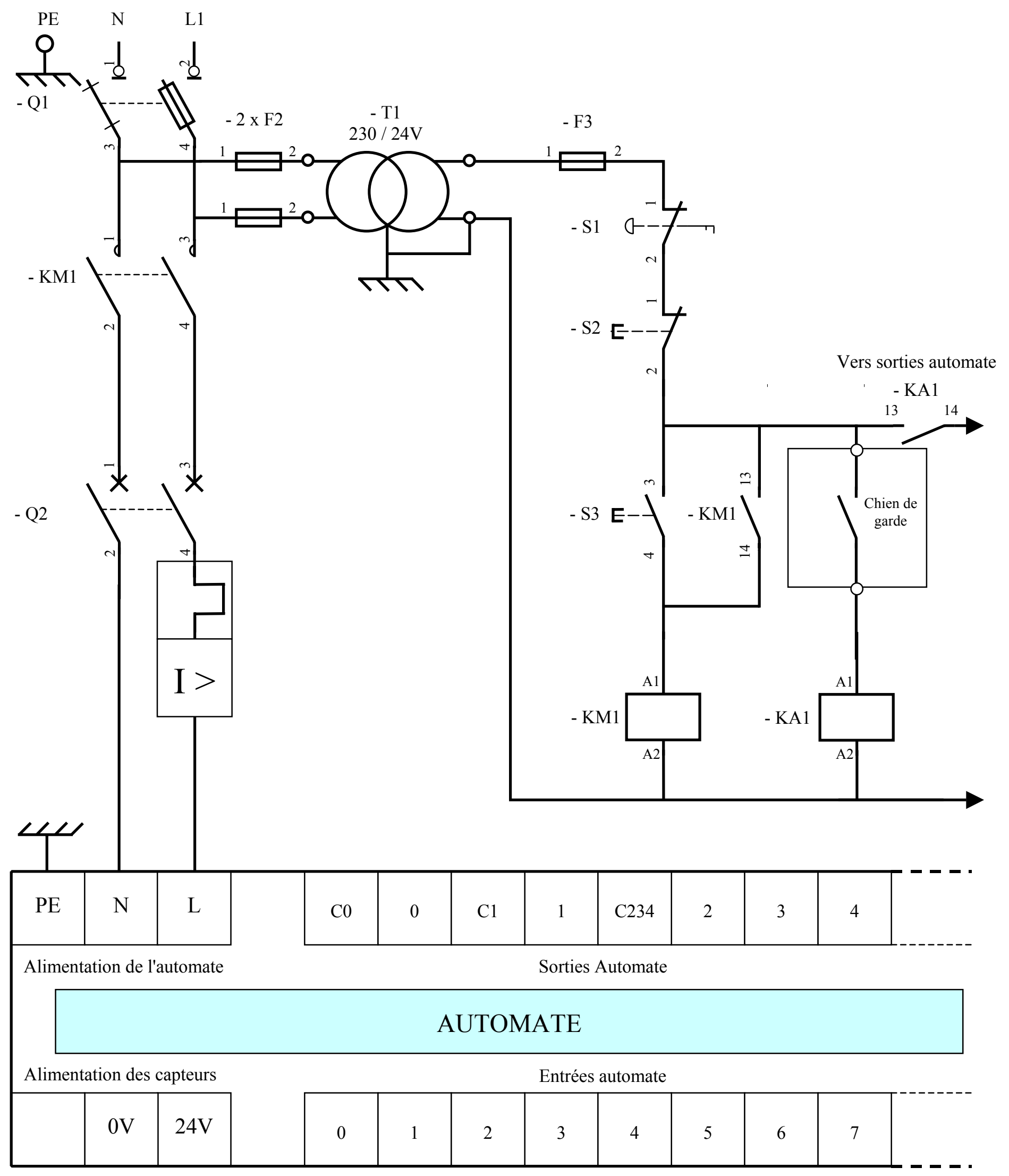




\section{b. Alimentation des entrées de l'automate:}

L'automate est pourvu généralement d'une alimentation pour les capteurs/détecteurs (attention au type de logique utilisée : logique positive ou négative).

Les entrées sont connectées au OV (commun) de cette alimentation.

Les informations des capteurs/détecteurs sont traitées par les interfaces d'entrées.

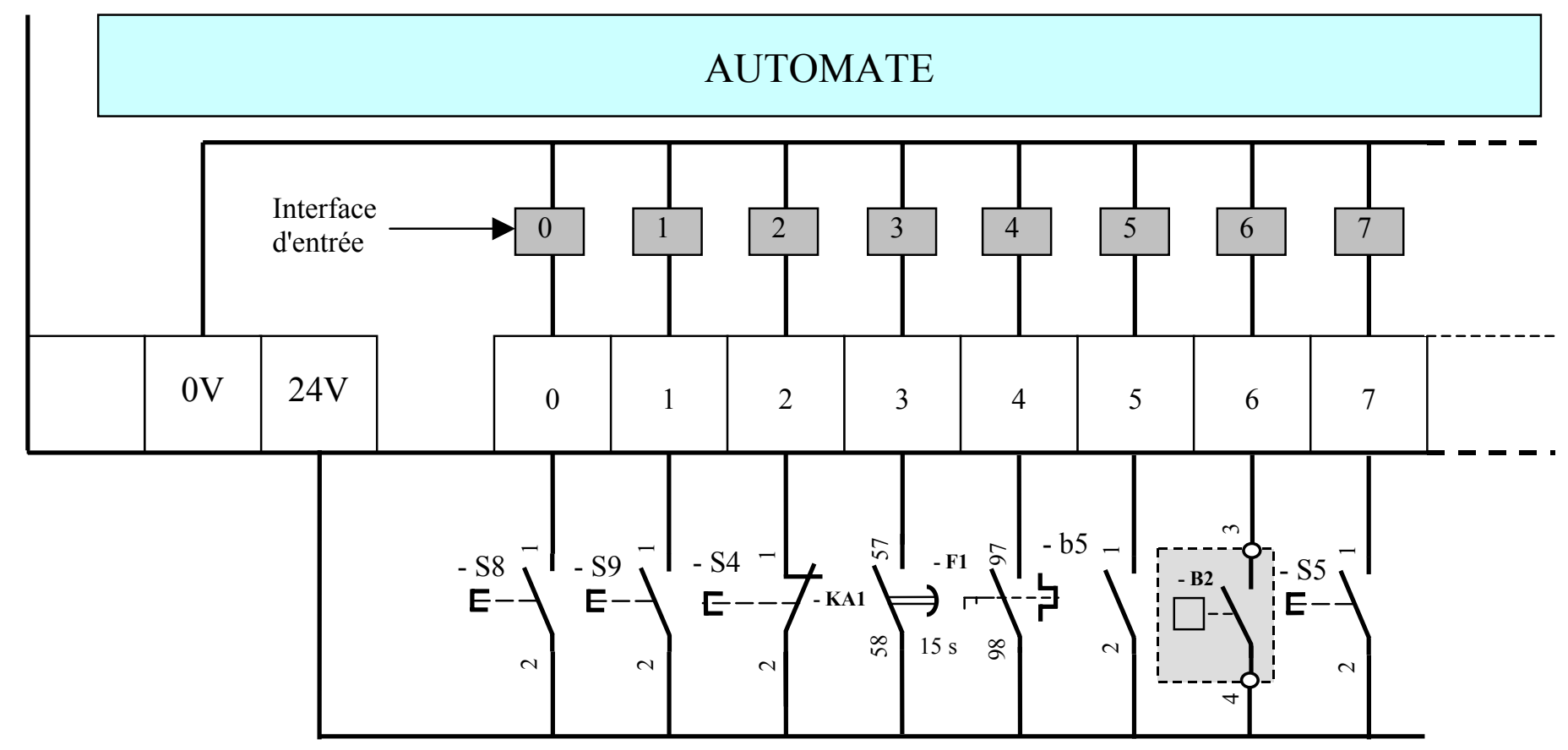

\section{c. Alimentation des sorties de l'automate :}

Les interfaces de sorties permettent d'alimenter les divers préactionneurs.

Il est souhaitable d'équiper chaque préactionneur à base de relais de circuits $\mathrm{RC}$ (non représentés).

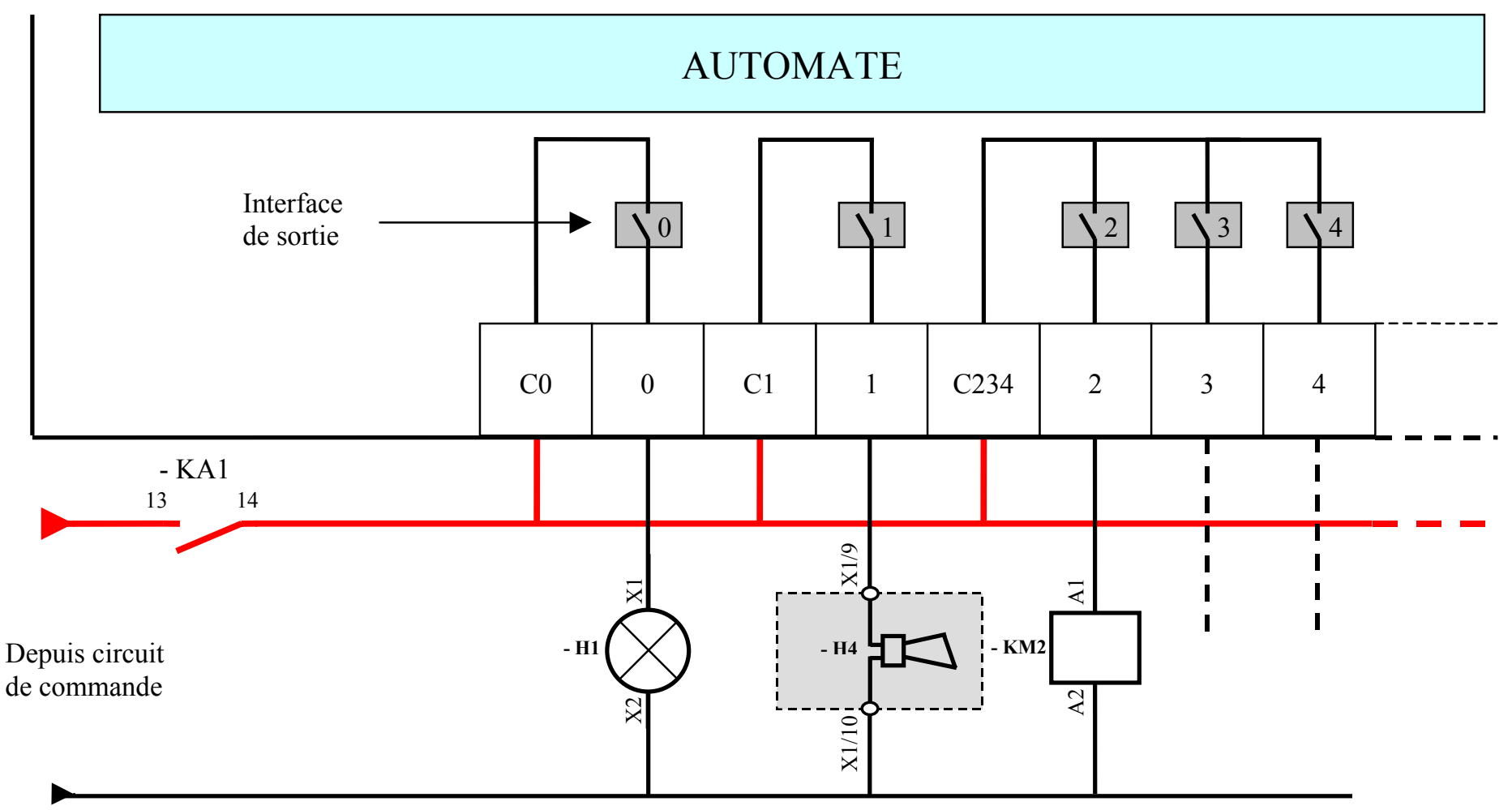




\section{Traitement du programme automate :}

Tous les automates fonctionnent selon le même mode opératoire :

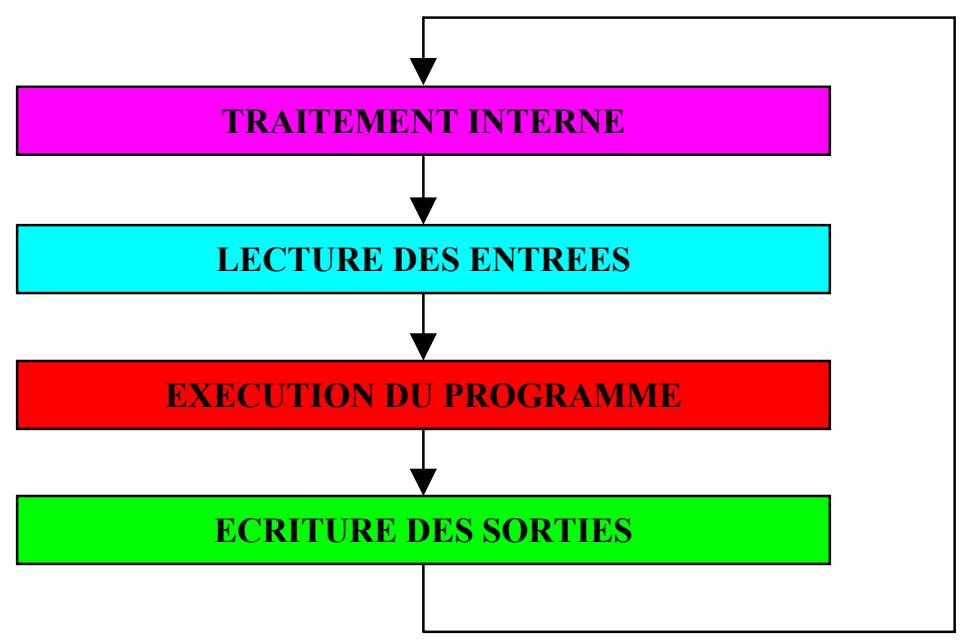

Traitement interne : L'automate effectue des opérations de contrôle et met à jour certains paramètres systèmes (détection des passages en RUN / STOP, mises à jour des valeurs de l'horodateur, ...).

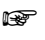

Lecture des entrées: L'automate lit les entrées (de façon synchrone) et les recopie dans la mémoire image des entrées.

Exécution du programme : L'automate exécute le programme instruction par instruction et écrit les sorties dans la mémoire image des sorties.

Ecriture des sorties: L'automate bascule les différentes sorties (de façon synchrone) aux positions définies dans la mémoire image des sorties.

Ces quatre opérations sont effectuées continuellement par l'automate (fonctionnement cyclique).

On appelle scrutation l'ensemble des quatre opérations réalisées par l'automate et le temps de scrutation est le temps mis par l'automate pour traiter la même partie de programme. Ce temps est de l'ordre de la dizaine de millisecondes pour les applications standards.

Le temps de réponse total (TRT) est le temps qui s'écoule entre le changement d'état d'une entrée et le changement d'état de la sortie correspondante :

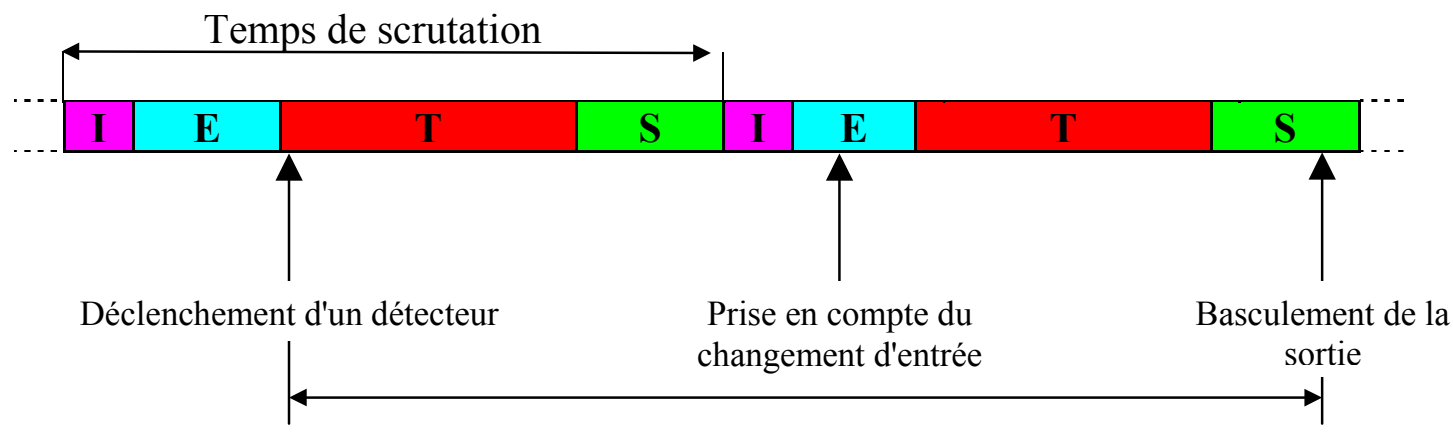

Temps de réponse total

Le temps de réponse total est au plus égal à deux fois le temps de scrutation (sans traitement particulier). 
Le temps de scrutation est directement lié au programme implanté. Ce temps peut être fixé à une valeur précise (fonctionnement périodique), le système indiquera alors tout dépassement de période.

Dans certains cas, on ne peut admettre un temps de réponse aussi long pour certaines entrées : ces entrées pourront alors être traitées par l'automate comme des événements (traitement événementiel) et prises en compte en priorité (exemples : problème de sécurité, coupure d'alimentation ...).

Certains automates sont également pourvues d'entrées rapides qui sont prises en compte avant le traitement séquentiel mais le traitement évènementiel reste prioritaire.

\section{Exemple:}

Les automates TSX micro (Télémécanique) offrent deux types de structure logicielle :

- Une structure monotâche :

Le traitement se fait de la façon décrite au début de ce chapitre. Le programme n'est alors lié qu'à une seule tâche : la tâche maître.

- Une structure multitâche :

A la tâche précédente peut être rajouté deux autres tâches : la tâche rapide et la tâche événementielle. La tâche rapide est alors périodique pour laisser le temps à la tâche maître de s'exécuter (la moins prioritaire). La tâche événementielle est prioritaire sur les autres tâches.

\section{Exemple de traitement multitâche:}

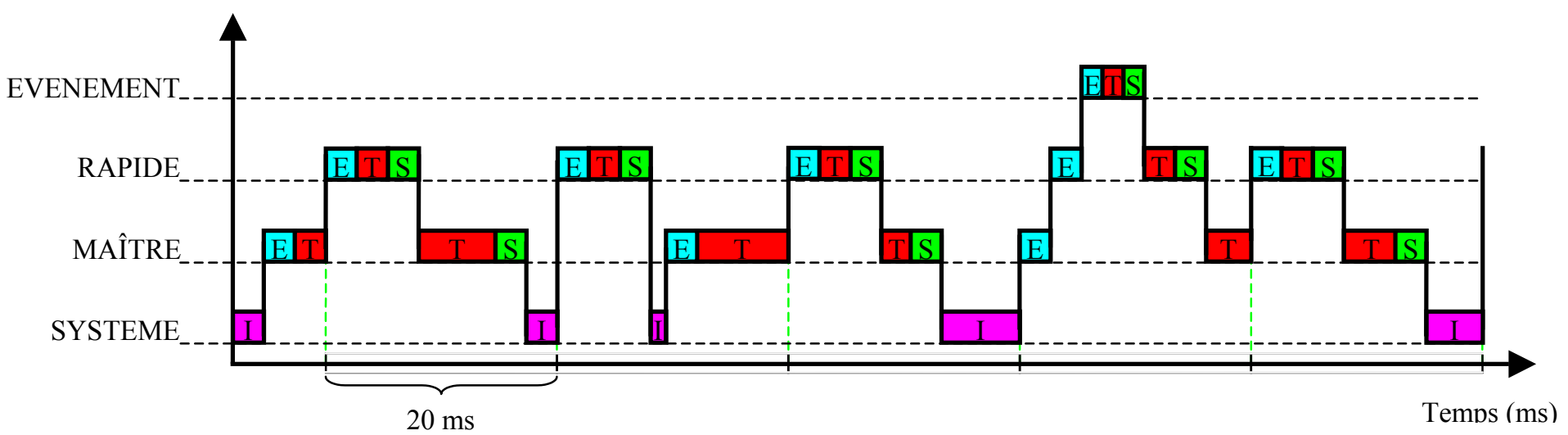

La périodicité de la tâche rapide est ici fixée à $20 \mathrm{~ms}$.

Il faudra veiller aux temps de cycle de la tâche maître. 


\section{Programmation :}

\section{a. Langages de programmation :}

Il existe 4 langages de programmation des automates qui sont normalisés au plan mondial par la norme CEI 61131-3.

Chaque automate se programmant via une console de programmation propriétaire ou par un ordinateur équipé du logiciel constructeur spécifique.

- Liste d'instructions (IL : Instruction list) : Langage textuel de même nature que l'assembleur (programmation des microcontrôleurs).

Très peu utilisé par les automaticiens.

\begin{tabular}{|c|c|c|}
\hline ! 舛 Lo: & $\begin{array}{l}\text { LD } \\
\text { ANDN } \\
\text { ORi } \\
\text { AND } \\
\text { jond } \\
\text { ST }\end{array}$ & 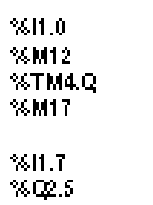 \\
\hline ! ' \% & $\begin{array}{l}\text { LD } \\
\text { ANDN } \\
\text { aNDN } \\
\text { IN } \\
\text { LD } \\
\text { aND } \\
\text { aND } \\
\text { [SMUNH := }\end{array}$ & 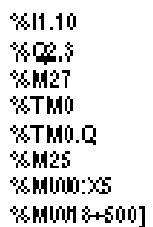 \\
\hline
\end{tabular}

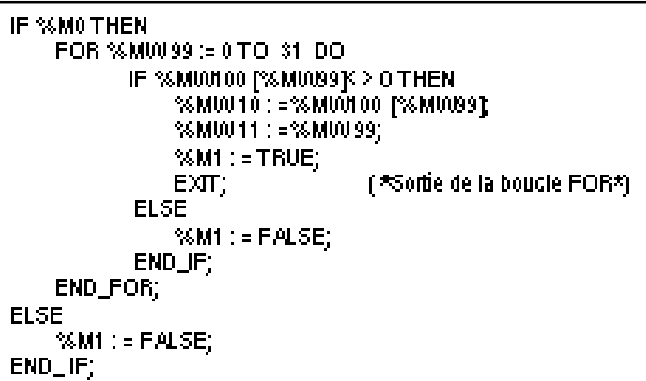$$
\text { ELSE }
$$

Peu utilisé par les automaticiens.

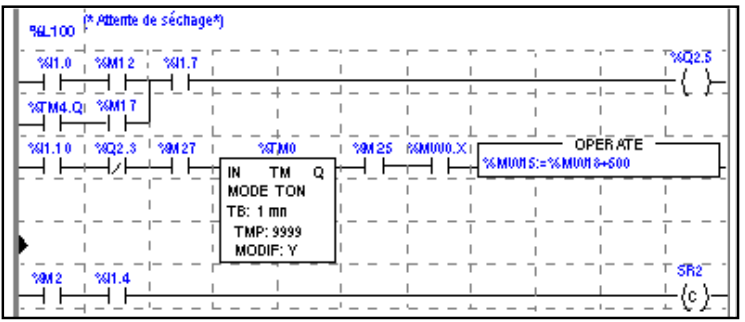

C'est le plus utilisé.

- Blocs Fonctionnels (FBD : Function Bloc Diagram): Langage graphique ou des fonctions sont représentées par des rectangles avec les entrées à gauche et les sorties à droites. Les blocs sont programmés (bibliothèque) ou programmables. Utilisé par les automaticiens.

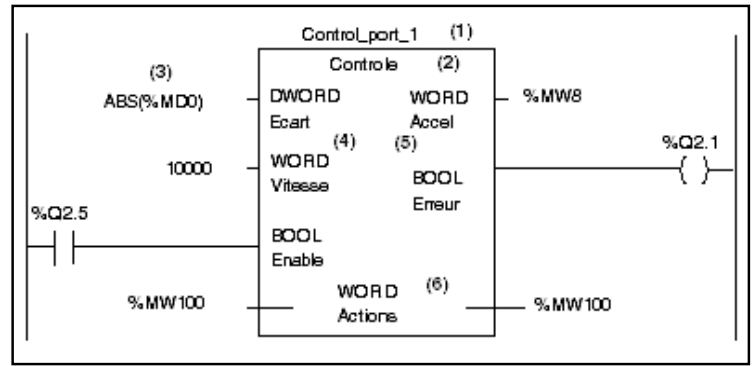




\section{b. Programmation à l'aide du GRAFCET (SFC: Sequential Function Chart) :}

Le GRAFCET, langage de spécification, est utilisé par certains constructeurs d'automate (Schneider, Siemens ) pour la programmation. Parfois associé à un langage de programmation, il permet une programmation aisée des systèmes séquentiels tout en facilitant la mise au point des programmes ainsi que le dépannage des systèmes. On peut également traduire un grafcet en langage en contacts et l'implanter sur tout type d'automate.

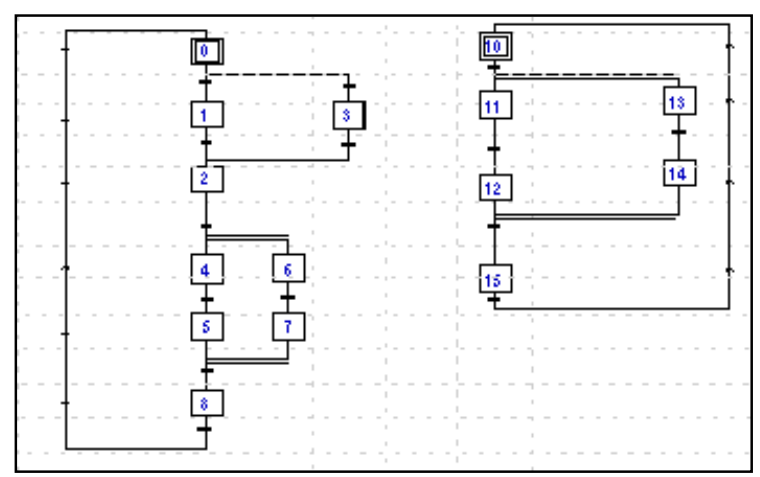

Certains logiciels permettent une programmation totale en langage GRAFCET et permettent de s'adapter à la plupart des automates existants (logiciels CADEPA ou AUTOMGEN).

\section{VII.Sécurité :}

Les systèmes automatisés sont, par nature, source de nombreux dangers (tensions utilisées, déplacements mécaniques, jets de matière sous pression ...).

Placé au cœur du système automatisé, l'automate se doit d'être un élément fiable car :

- un dysfonctionnement de celui-ci pourrait avoir de graves répercussions sur la sécurité des personnes,

- les coûts de réparation de l'outil de production sont généralement très élevé,

- un arrêt de la production peut avoir de lourdes conséquences sur le plan financier.

Aussi, l'automate fait l'objet de nombreuses dispositions pour assurer la sécurité :

- Contraintes extérieures : l'automate est conçu pour supporter les différentes contraintes du monde industriel et à fait l'objet de nombreux tests normalisés (tenue aux vibrations, CEM ...)

- Coupures d'alimentation : l'automate est conçu pour supporter les coupures d'alimentation et permet, par programme, d'assurer un fonctionnement correct lors de la réalimentation (reprises à froid ou à chaud)

- Mode RUN/STOP : Seul un technicien peut mettre en marche ou arrêter un automate et la remise en marche se fait par une procédure d'initialisation (programmée)

- Contrôles cycliques:

Procédures d'autocontrôle des mémoires, de l'horloges, de la batterie, de la tensions d'alimentation et des entrées / sorties

Vérification du temps de scrutation à chaque cycle appelée Watchdog (chien de garde), et enclenchement d'une procédure d'alarme en cas de dépassement de celui-ci (réglé par l'utilisateur) 
- Visualisation : Les automates offrent un écran de visualisation où l'on peut voir l'évolution des entrées / sorties

La défaillance d'un automate programmable pouvant avoir de graves répercussions en matière de sécurité, les normes interdisent la gestion des arrêts d'urgence par l'automate ; celle-ci doit être réalisée en technologie câblée.

On peut également ajouter des modules de sécurité à l'automate (sécurité des machines).

Il existe enfin des automates dits de sécurité (APIdS) qui intègrent des fonctions de surveillance et de redondance accrues et garantissent la sécurité des matériels.

\section{VIII.Les automates et la communication :}

Avec le développement des systèmes automatisés et de l'électronique (circuits ASICs), la recherche de la baisse des coûts et la nécessité actuelle de pouvoir gérer au mieux la production, c'est à dire :

- de recevoir les données liées à une application le plus rapidement possible,

- de consulter, contrôler ou de modifier les paramètres d'une application à distance ;

sont apparues de nouvelles technologies de câblage et de communications entre les divers constituants des automatismes.

\section{a. Les bus de terrain :}

Pour diminuer les coûts de câblage des entrées / sorties des automates (systèmes étendus), sont apparus les bus de terrains. L'utilisation de blocs d'entrées / sorties déportés à permis tout d'abord de répondre à cette exigence.

\section{$\underline{\text { Avant : }}$}

Les capteurs / préactionneurs distants impliquaient de grandes longueurs de câbles.

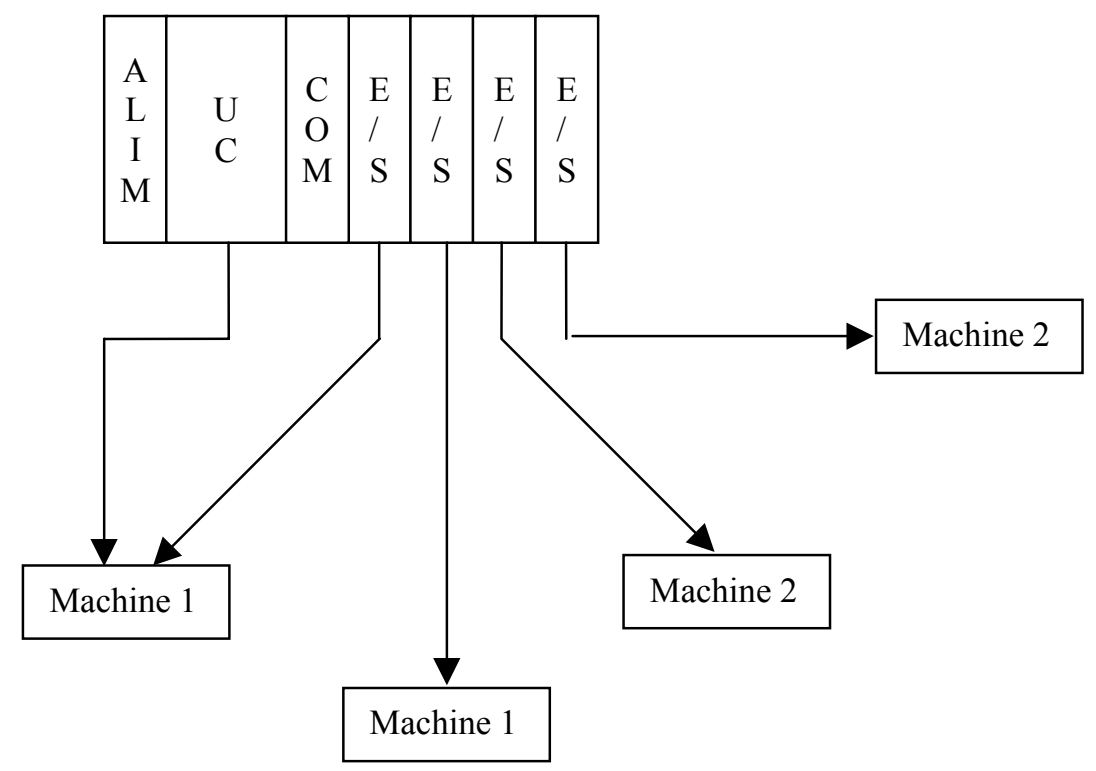




\section{$\underline{1 \text { ère évolution : }}$}

Les interfaces d'entrées/ sorties sont déportées au plus près des capteurs.

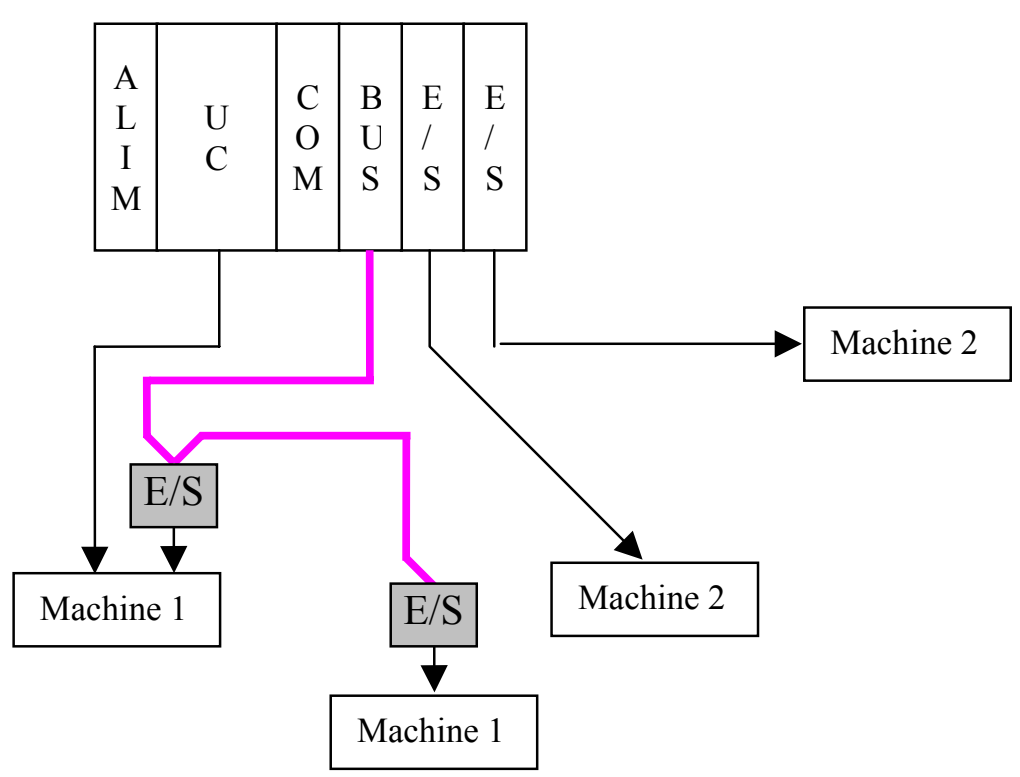

Avec l'avènement des ASICs, les capteurs, détecteurs ... sont devenus "intelligents" et ont permis de se connecter directement au bus (médium).

\section{Aujourd'hui :}

Les capteurs et les préactionneurs "intelligents" (IHM, variateurs, distributeurs ...) permettent la connexion directe au bus.

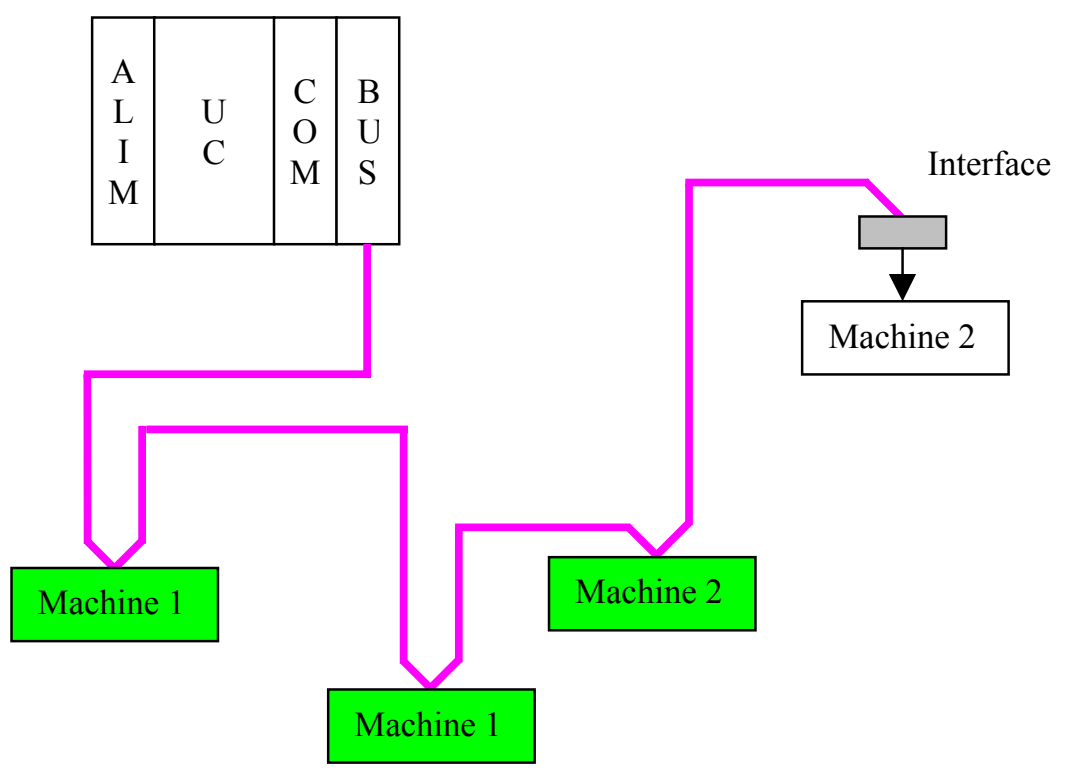

Pour assurer le "multiplexage" de toutes les informations en provenance des capteurs / préactionneurs ont été développés plusieurs protocoles de communication et des standards sont apparus (normalisés ou standards de fait).

\section{Exemple :}

Le bus ASi (Actuators Sensors interface) est un bus de capteurs/actionneurs de type Maître / Esclave qui permet de raccorder 31 esclaves (capteurs ou préactionneurs) sur un câble spécifique (deux fils) transportant les données et la puissance.

Ce bus est totalement standardisé et permet d'utiliser des technologies de plusieurs constructeurs (interopérabilité). L'automate est pour cela doté d'un coupleur ASi. 


\section{$\underline{\text { Avantages des bus de terrain : }}$}

Réduction des coûts de câblage et possibilité de réutiliser le matériel existant

Réduction des coûts de maintenance

Possibilités de communication

Inconvénients des bus de terrain :

Taille du réseau limitée

Adaptabilité aux applications à temps critique

Coût global

Autres bus de terrain : Batibus (norme EIB), Interbus-S, CANopen

\section{b. Les réseaux de terrain :}

L'émergence de ces nouvelles technologies à conduit à la définition de plusieurs catégories de réseaux locaux industriels (pyramide CIM) :

- $\quad$ les réseaux de terrain,

- $\quad$ les réseaux de cellule,

- $\quad$ les réseaux de supervision et de commande

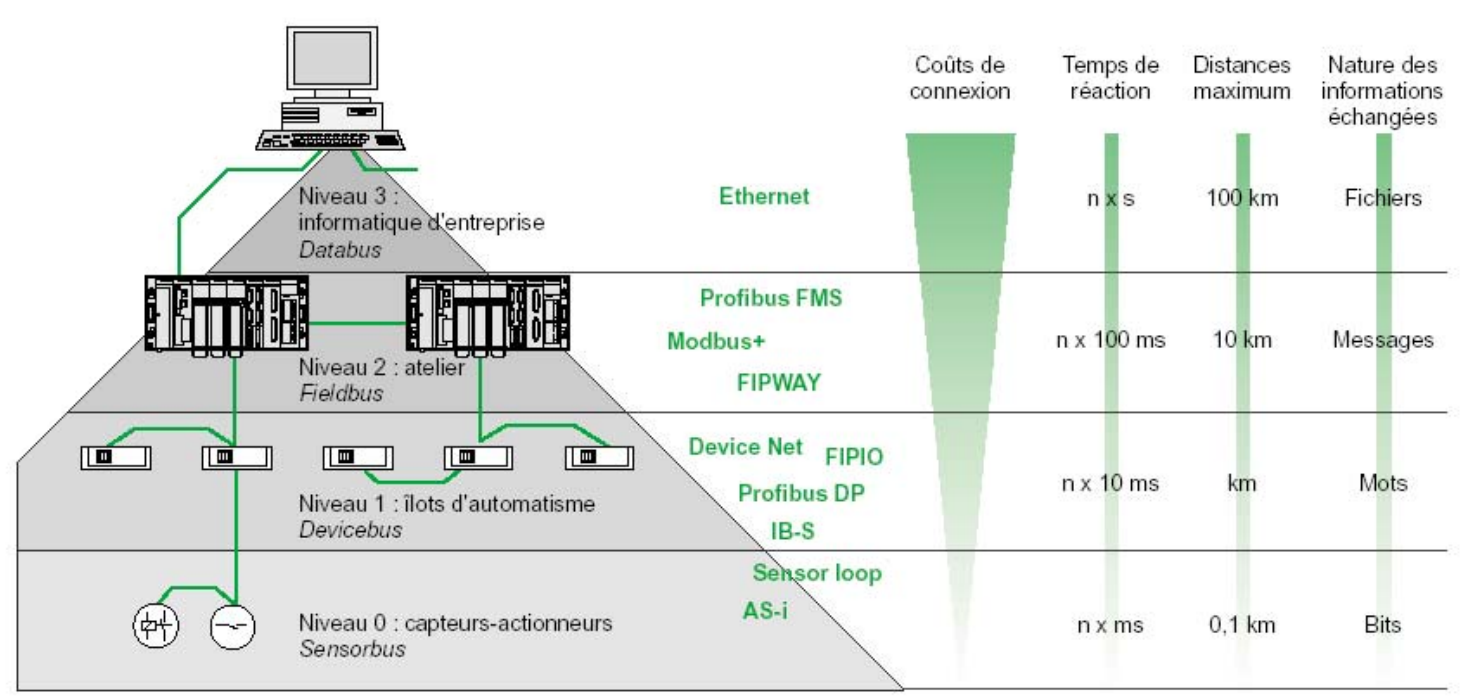

La nécessité de communication entre cellules (communication entre automates) a permis de voir apparaître de nombreuses normes de communication (Profibus, Fip ...).

Le déterminisme nécessaire pour certaines applications conduit à l'utilisation de réseaux Maître / Esclave. Au niveau de l'entreprise, le temps n'est plus critique et la norme Ethernet à pu se développer rapidement, permettant ainsi la visualisation et la commande des process via le réseau Internet.

La tendance actuelle est à l'introduction des réseaux Ethernet au plus près des automatismes (exemple : norme Profinet). 


\section{Critères de choix d'un automate:}

Le choix d'un automate programmable est en premier lieu le choix d'une société ou d'un groupe et les contacts commerciaux et expériences vécues sont déjà un point de départ.

Les grandes sociétés privilégieront deux fabricants pour faire jouer la concurrence et pouvoir "se retourner" en cas de "perte de vitesse" de l'une d'entre elles.

Le personnel de maintenance doit toutefois être formé sur ces matériels et une trop grande diversité des matériels peut avoir de graves répercussions. Un automate utilisant des langages de programmation de type GRAFCET est également préférable pour assurer les mises au point et dépannages dans les meilleures conditions.

La possession d'un logiciel de programmation est aussi source d'économies (achat du logiciel et formation du personnel). Des outils permettant une simulation des programmes sont également souhaitables.

Il faut ensuite quantifier les besoins :

- Nombre d'entrées / sorties : le nombre de cartes peut avoir une incidence sur le nombre de racks dès que le nombre d'entrées / sorties nécessaires devient élevé.

- Type de processeur : la taille mémoire, la vitesse de traitement et les fonctions spéciales offertes par le processeur permettront le choix dans la gamme souvent très étendue.

- Fonctions ou modules spéciaux : certaines cartes (commande d'axe, pesage ...) permettront de "soulager" le processeur et devront offrir les caractéristiques souhaitées (résolution, ...).

- Fonctions de communication : l'automate doit pouvoir communiquer avec les autres systèmes de commande (API, supervision ...) et offrir des possibilités de communication avec des standards normalisés (Profibus ...). 